\title{
Análisis morfométrico y molecular (ADNmt) de abejas melíferas (Apis mellifera L.) en el estado de Tabasco, México
}

Juan Florencio Gómez Leyva ${ }^{\text {a }}$

Omar Argüello Nájera b

Pablo Jorge Vázquez Encino ${ }^{c}$

Luis Ulises Hernández Hernández ${ }^{\text {d }}$

Emeterio Payró de la Cruz ${ }^{\mathrm{c} *}$

a Tecnológico Nacional de México. Campus Instituto Tecnológico de Tlajomulco Jalisco, Laboratorio de Biología Molecular. Km 10 Carretera a San Miguel Cuyutlán. Tlajomulco de Zúñiga, Jalisco, México.

${ }^{\mathrm{b}}$ El Colegio de la Frontera Sur (ECOSUR-San Cristóbal, Chiapas). San Cristóbal L.C. Chiapas. México.

c Tecnológico Nacional de México. Campus Instituto Tecnológico de la Zona Olmeca. Laboratorio de Biología. Zaragoza s/n. Villa Ocuiltzapotlán, Centro, Tabasco, México.

${ }^{\text {d} U n i v e r s i d a d ~ J u a ́ r e z ~ A u t o ́ n o m a ~ d e ~ T a b a s c o . ~ D i v i s i o ́ n ~ A c a d e ́ m i c a ~ d e ~ C i e n c i a s ~ A g r o p e c u a r i a s . ~}$ Tabasco. México.

*Autor de correspondencia: epayro@itzonaolmeca.edu.mx

\section{Resumen:}

La apicultura en México se sostiene con subespecies de abejas (Apis mellifera L) europeas y africanizadas. Debido a la dificultad en la diferenciación morfológica de las poblaciones Europeas (E) y Africanizadas (A) de A. mellifera, el objetivo de este trabajo fue realizar un análisis comparativo entre la técnica morfométrica FABIS, contra el diagnóstico empleando el polimorfismo en la longitud de los fragmentos de restricción (RFLP) del ADN 
mitocondrial (ADNmt). Se emplearon muestras de abejas procedentes de 135 colonias comerciales (CC), 15 colonias pie de cría (CPC) y 3 colonias silvestres (CS) localizados en diferentes municipios del estado de Tabasco $(\mathrm{N}=153)$. Ambos métodos de diagnóstico, identificaron a las CPC como europeas y las CS como africanizadas, pero en las CC el método FABIS no pudo definir 9 colonias, dándolas como sospechosas (S) y otras 50 no coincidieron con el resultado del método molecular, por lo que, en total ambos métodos coincidieron en 94 identificaciones (61.44\%). El agrupamiento bayesiano basado en el análisis de las doce variables morfométricas mostró que las categorías A-CC y E-CC, forman un grupo cercano a la categoría E-CPC; mientras que la categoría A-CS presentó la mayor distancia formando un grupo aislado. Por lo tanto, abejas de CC tienen características morfométricas tendientes a abejas E-CPC. Este trabajo también pretende ser una contribución a la falta de registros sobre la africanización en el estado de Tabasco. Se recomienda emplear el método molecular para discriminar entre abejas E/A, por no estar afectado por los factores ambientales.

Palabras clave: Colonias, Africanización, PCR, Morfometría, ADNmt.

Recibido: $12 / 11 / 2018$

Aceptado: 08/04/2021

\section{Introducción}

Existen al menos 24 subespecies de Apis mellifera agrupadas en cuatro ramas evolutivas principales: Línea $\mathrm{O}$ (Oriente próximo), línea A (Africana), línea M (Mediterráneo occidental) y línea C (Mediterráneo central y sureste de Europa) ${ }^{(1)}$. En 1956, con el objetivo de estudiar la adaptación a los climas tropicales y mejorar la producción de miel, abejas reinas africanas (A. mellifera scutellata) y sus híbridos fueron importadas de Sudáfrica a Brasil ${ }^{(2,3)}$. En 1957 el escape de las abejas en Brasil, dio lugar al cruzamiento masivo con las abejas locales de origen europeo, lo cual generó poblaciones con genotipos híbridos denominadas africanizadas $^{(4)}$. En México la abeja africanizada fue primeramente identificada en septiembre de 1986, cuando un enjambre fue atrapado e identificado cerca de Tapachula, Chiapas, en la zona fronteriza con Guatemala ${ }^{(5,6)}$. Posteriormente se reportó la presencia de abejas africanizadas colectadas en 1987 en los municipios de Tenosique y Centla, Tabasco, así como en los municipios de Coatzacoalcos Veracruz, Palenque Chiapas y Hopelchen Campeche $^{(7)}$. En la península de Yucatán, las abejas silvestres africanizadas (descendientes de A. mellifera scutellata), fueron reportadas en $1987^{(8)}$. Diversos estudios han demostrado que la abeja Apis mellifera scutellata posee cualidades que merecen destacarse; 
principalmente son productoras de miel en climas templados, toleran ambientes fríos, son prolíficas padeciendo menos enfermedades por parasitosis que otras $\operatorname{razas}^{(9)}$. Las abejas africanizadas tienden a recolectar más polen y más propóleos que las abejas europeas ${ }^{(10)}$. Debido a su mayor capacidad de adaptación al medio ambiente tropical, se encuentran ampliamente distribuidas en el continente americano $^{(11)}$.

De acuerdo con FAOSTAT ${ }^{(12)}$ hasta 2019, China generó la mayor producción y exportación de miel a nivel mundial, con 120,845 t exportadas, seguido de India (65,351 t exportadas), Argentina (63,522 t), Ucrania (54,834 t), Brasil (30,039 t), Alemania (25,239 t) y México $(25,122$ t). En México, durante el periodo de enero a noviembre de 2020 las exportaciones de miel alcanzaron las 26,077 t, lo que significó un aumento de la demanda en un $3.66 \%$, comparado con el año 2019. El 62.86 \% de la producción de miel (toneladas) en 2020, se concentró en siete estados, entre los que destacaron Jalisco $(6,059)$, Yucatán $(5,529)$, Chiapas $(5,434)$, Campeche $(5,375)$, Veracruz $(4,645)$, Oaxaca $(4,533)$ y Puebla $(2,450)$. Tabasco sólo reportó una producción de 405 t, ocupando el lugar 25, como productor de miel a nivel nacional, muy inferior a sus estados vecinos ${ }^{(13)}$, a pesar de disponer de recursos botánicos para el desarrollo de la apicultura. Estos datos reflejan la magnitud de la problemática de la apicultura en esta entidad ubicada en el trópico húmedo mexicano.

Actualmente la apicultura en México se practica con abejas africanizadas y diversas subespecies de abejas europeas que fueron introducidas a México tales como Apis mellifera mellifera y Apis mellifera ligustica. En el estado de Tabasco, los genotipos de abejas que coexisten, mediante cruzamientos naturales sucesivos, han generado un pool genético de abejas africanizadas del que no se cuenta con suficientes reportes que permitan valorar su dinámica poblacional y sus efectos en la productividad. Una limitante es que son muy difíciles de identificar por sus características morfométricas externas ${ }^{(9)}$, sin embargo existen diversas técnicas moleculares ${ }^{(14,15)}$; las cuales se han utilizado exitosamente en diversos estudios relativos a la estructura, diversidad genética y filogenia, determinación de mitotipos y flujo genético de las poblaciones de abejas ${ }^{(16-22)}$.

En el presente estudio se hizo un análisis comparativo entre la técnica morfométrica FABIS (por sus siglas en inglés Fast Africanized Bee Identification System) y el diagnóstico RFLP (por sus siglas en inglés Restriction Fragment Length Polymorphism), empleando el polimorfismo en la longitud de los fragmentos de restricción del ADN mitocondrial (ADNmt) para identificación de abejas africanizadas del estado de Tabasco y su relación con 12 variables morfométricas alares, femorales y abdominales. 


\section{Material y métodos}

El estado de Tabasco se encuentra ubicado al sureste de la República Mexicana, en la región del trópico húmedo entre los $17^{\circ} 15^{\prime} 00^{\prime \prime}-18^{\circ} 39^{\prime} 07^{\prime \prime} \mathrm{N}$ y los $90^{\circ} 50^{\prime} 23^{\prime \prime}-94^{\circ} 07^{\prime} 49^{\prime \prime} \mathrm{O}^{(23)}$.

\section{Material biológico}

Se obtuvieron muestras de abejas obreras tomadas al azar de tres colonias por apiario. Se colectaron directamente de la cámara de cría aproximadamente 400 abejas obreras por colonia, las cuales se colocaron en recipientes etiquetados conteniendo etanol al $96 \%$ y conservadas hasta su uso a $-20^{\circ} \mathrm{C}$. El presente estudio comprende el muestreo de 135 colonias de abejas comerciales (CC) de productores cooperantes, 15 colonias pie de cría (CPC) con abejas reinas inseminadas y 3 colonias silvestres (CS). Los análisis morfométricos y los análisis moleculares se realizaron, en las siguientes etapas:

\section{Análisis morfométrico}

De cada muestra de las colonias, se tomaron 10 abejas obreras cuyas estructuras fueron disectadas y fijadas en porta objetos para su observación y análisis morfométrico. Las estructuras se digitalizaron con un equipo de microscopía Marca Karl Zeiss con cámara integrada y el software, Axiovision LE 472. Las variables de longitud y ancho se midieron en milímetros (mm): Longitud del ala anterior derecha (V1 LAAD), ancho del ala anterior derecha (V2 AAAD), longitud del ala posterior derecha (V3 LAPD), número de hámulus del ala posterior (V4 NHAP), longitud de la trompa (V5 LTR), longitud de la tibia pata posterior (V6 LTPP), longitud del fémur pata posterior (V7 LFPP), ancho del cuarto tergito (V8 ACT), longitud del cuarto tergito (V9 LCT), longitud de la banda del cuarto tergito (V10 LBCT), ancho del cuarto esternito (V11 ACE), y longitud del cuarto esternito (V12 LCE). Para la identificación de abejas africanizadas se usó el método FABIS (Por sus siglas en inglés Fast Africanized Bee Identification Sistem) ${ }^{(22,24)}$. Para determinar el índice de africanización por subregiones geográficas, también se calcularon las frecuencias de los morfotipos. 


\section{Extracción de ADN}

La extracción de ADN se realizó empleando el método descrito por Doyle y Doyle ${ }^{(16)}$ modificado: se depositaron cinco abejas obreras en un mortero, adicionando amortiguador de extracción (Tris- $\mathrm{HCl} 100 \mathrm{mM}, \mathrm{NaCl} 1.5 \mathrm{M}$, EDTA $20 \mathrm{mM} \mathrm{pH} \mathrm{8,} \mathrm{CTAB} 4 \%$, PVP $40.4 \%$, ácido ascórbico $0.1 \%, \beta$-mercaptoetanol $0.3 \%$ ) precalentado, recuperando la fase acuosa en tubos cónicos. Se recuperan $500 \mu \mathrm{l}$ de la fase acuosa, se incubaron a $60{ }^{\circ} \mathrm{C}$ en baño María durante 1 h y se agitó cada 15 min; se dejó reposar hasta llegar a temperatura ambiente. Se agregaron $500 \mu \mathrm{l}$ de cloroformo: alcohol isoamílico $(49: 1 \mathrm{v} / \mathrm{v})$. El tubo se agitó hasta mezclar y formar una emulsión. Se centrifugó a 14,000 rpm durante 5 min y la fase acuosa se transfirió a un tubo nuevo con una micropipeta; se agregó un volumen de isopropanol frío y se dejó incubar a $-20{ }^{\circ} \mathrm{C}$ durante $15 \mathrm{~min}$. Se centrifugó a 5,000 rpm durante $5 \mathrm{~min}$ y el sobrenadante fue descartado. La pastilla obtenida se lavó con etanol frío al $70 \%$, se agitó para lavarla completamente, se centrifugó a 14,000 rpm durante $2 \mathrm{~min}$; retirando el etanol y dejando secar a temperatura ambiente. Finalmente, la pastilla se suspendió en $200 \mu \mathrm{l}$ de agua inyectable y se almacenó a $20^{\circ} \mathrm{C}$. El ADN extraído se observó en un gel de agarosa al $1 \%$ y se cuantificó por absorbancia a $260 \mathrm{~nm}$.

\section{Amplificación por PCR del ADN mitocondrial.}

La región de 485 pb del gen citocromo b, fue amplificada usando los oligonucleotidos CytbA-

F (5' TATGTACTACCATGAGGACAAATATC) y CytbA-R (5' ATTACACCTCCTAATTTATTAGGAAT). Se empleó un equipo termociclador Mca. Genius, modelo Techne, programando las condiciones de corrida: $94{ }^{\circ} \mathrm{C}$ ( $2 \mathrm{~min}$ ), seguida por 30 ciclos $94^{\circ} \mathrm{C}(1 \mathrm{~min}), 50{ }^{\circ} \mathrm{C}(1 \mathrm{~min})$ y $72^{\circ} \mathrm{C}(1 \mathrm{~min})$, después del ciclo final $72{ }^{\circ} \mathrm{C}(7 \mathrm{~min})$.

\section{Análisis en la longitud de los fragmentos de restricción (RFLP)}

Después de la amplificación de las muestras, $10 \mu \mathrm{l}$ de los productos de PCR fueron digeridos con $1 \mathrm{U}$ de enzima de restricción Bgl II (Invitrogen), a $37{ }^{\circ} \mathrm{C}$ durante 4 h. La digestión se sometió a electroforesis en un gel de agarosa $2 \%$, visualizada bajo luz ultravioleta. Los sitios de restricción se midieron como: Mitotipo europeo (E), al visualizar un patrón de dos fragmentos (194 y $291 \mathrm{pb}$ ), o mitotipo africanizado (A), al visualizar un fragmento único sin digerir de $485 \mathrm{pb}^{(1,17)}$. 
Cuando fue detectado el mitotipo, se clasificó en cuatro categorías: A-CS (Mitotipo africanizado, colonia silvestre); A-CC (Mitotipo africanizado, colonia comercial); E-CC (Mitotipo europeo, colonia comercial) y E-CPC (Mitotipo europeo, colonia pie de cría). Tomando como fuentes de variación las categorías anteriormente descritas, los datos morfométricos de las abejas se sometieron a análisis de varianza y prueba de medias Bonferroni $(P \leq 0.05 ; 95 \%$ de confianza) cuando fue requerido. El análisis multivariado se realizó mediante componentes principales, análisis discriminante y de clúster para los atributos de los grupos, y como aporte de cada una de las variables en función de las distancias de enlace, se empleó el software estadístico Statgraphics centurión XVI, Versión 2016 e INFOSTAT.

\section{Resultados}

Como puede observarse en el Cuadro 1, se encontraron diferencias estadísticas significativas entre las subregiones en 7 de las 12 variables morfométricas estudiadas (V1 LAAD, V3 LAPD, V5 LTR, V8 ACT, V9 LCT, V10 LBCT y V12 LCE). Las abejas de la subregión Chontalpa en promedio mostraron los valores más grandes en la mayoría de las variables, excepto en la V6 LTPP $(P=0.9298)$, ya que el valor más grande se encontró en la subregión Centro $(3.02 \mathrm{~mm})$, con una diferencia de 0.01 y $0.03 \mathrm{~mm}$ respecto a las otras subregiones. No se encontraron diferencias estadísticamente significativas entre las subregiones respecto a esta variable, así como en la V2 AAAD $(P=0.1368)$, V4 NHAP $(P=0.2764)$, V7 LFPP $(P=0.0945)$ y V11 ACE (0.5569). El análisis de componentes principales mostró que tres componentes tienen un valor propio (Eigenvalue) mayor o igual a 1.0, los cuales en conjunto explican el $60.088 \%$ de la variabilidad de los datos originales. La CP1, tiene una correlación positiva con todas las variables morfométricas estudiadas, mientras que la $\mathrm{CP} 2$, tiene una correlación positiva con cinco variables y negativa o nula con el resto. La CP3, tiene una correlación positiva con ocho variables, y negativa o nula con el resto. 
Cuadro 1: Resumen de 12 variables morfométricas medidas en 153 colonias del estado de Tabasco (mm)

\begin{tabular}{|c|c|c|c|c|c|c|c|c|c|c|c|c|c|c|}
\hline Subregión & n & Resumen & V1 & V2 & $\mathbf{V 3}$ & V4 & V5 & V6 & V7 & V8 & V9 & V10 & V11 & V12 \\
\hline \multirow[t]{4}{*}{ Centro } & 31 & Media & $9.02^{\mathrm{ab}}$ & 3.09 & $6.33^{\mathrm{ab}}$ & 20.88 & $4.98^{\mathrm{ab}}$ & 3.02 & 2.55 & $2.05^{\mathrm{ab}}$ & $8.82^{\mathrm{c}}$ & $7.23^{\mathrm{c}}$ & 1.60 & $3.99^{\mathrm{b}}$ \\
\hline & & D.E. & 0.11 & 0.06 & 0.10 & 0.870 & 0.37 & 0.07 & 0.07 & 0.08 & 0.21 & 0.30 & 0.05 & 0.14 \\
\hline & & Mín & 8.82 & 3.0 & 6.10 & 19.60 & 4.23 & 2.83 & 2.39 & 1.84 & 8.47 & 6.54 & 1.50 & 3.61 \\
\hline & & Máx & 9.25 & 3.32 & 6.56 & 23.80 & 5.57 & 3.19 & 2.67 & 2.36 & 9.23 & 8.15 & 1.72 & 4.27 \\
\hline \multirow[t]{4}{*}{ Chontalpa } & 68 & Media & $9.08^{\mathrm{a}}$ & 3.09 & $6.36^{\mathrm{a}}$ & 21.23 & $5.17^{\mathrm{a}}$ & 3.01 & 2.58 & $2.09^{\mathrm{a}}$ & $9.07^{\mathrm{a}}$ & $7.49^{\mathrm{b}}$ & 1.60 & $4.10^{\mathrm{a}}$ \\
\hline & & D.E. & 0.17 & 0.06 & 0.13 & 0.93 & 0.34 & 0.13 & 0.06 & 0.05 & 0.21 & 0.28 & 0.05 & 0.12 \\
\hline & & Mín & 8.64 & 2.94 & 5.99 & 18.40 & 4.24 & 2.10 & 2.48 & 1.93 & 8.63 & 6.83 & 1.48 & 3.83 \\
\hline & & Máx & 9.41 & 3.2 & 6.66 & 23.50 & 5.65 & 3.22 & 2.80 & 2.23 & 9.67 & 8.22 & 1.71 & 4.37 \\
\hline \multirow[t]{4}{*}{ Pantanos } & 24 & Media & $8.94^{\mathrm{b}}$ & 3.04 & $6.28^{\mathrm{b}}$ & 21.14 & $5.09^{\mathrm{a}}$ & 2.99 & 2.54 & $2.04^{\mathrm{b}}$ & $8.91^{\mathrm{bc}}$ & $7.32^{\mathrm{bc}}$ & 1.60 & $4.03^{\mathrm{ab}}$ \\
\hline & & D.E. & 0.13 & 0.05 & 0.12 & 0.880 & 0.35 & 0.06 & 0.05 & 0.03 & 0.15 & 0.33 & 0.05 & 0.09 \\
\hline & & Mín & 8.78 & 2.92 & 6.06 & 19.40 & 4.15 & 2.87 & 2.44 & 1.95 & 8.66 & 6.50 & 1.50 & 3.77 \\
\hline & & Máx & 9.24 & 3.13 & 6.59 & 22.80 & 5.47 & 3.16 & 2.65 & 2.09 & 9.24 & 7.92 & 1.70 & 4.15 \\
\hline \multirow[t]{4}{*}{ Ríos } & 12 & Media & $9.02^{\mathrm{ab}}$ & 3.11 & $6.31^{\mathrm{ab}}$ & 20.73 & $4.87^{\mathrm{ab}}$ & 3.01 & 2.57 & $2.06^{\mathrm{ab}}$ & $8.96^{\mathrm{abc}}$ & $7.51^{\mathrm{a}}$ & 1.58 & $4.06^{\mathrm{ab}}$ \\
\hline & & D.E. & 0.18 & 0.21 & 0.14 & 0.75 & 0.48 & 0.07 & 0.05 & 0.05 & 0.22 & 0.24 & 0.05 & 0.11 \\
\hline & & Mín & 8.81 & 2.98 & 6.14 & 19.70 & 4.35 & 2.89 & 2.50 & 1.97 & 8.54 & 7.13 & 1.53 & 3.87 \\
\hline & & Máx & 9.49 & 3.76 & 6.64 & 22.40 & 5.77 & 3.15 & 2.65 & 2.15 & 9.25 & 7.87 & 1.68 & 4.26 \\
\hline \multirow[t]{5}{*}{ Sierra } & 18 & Media & $9.05^{\mathrm{ab}}$ & 3.09 & $6.35^{\mathrm{ab}}$ & 21.08 & $4.73^{\mathrm{b}}$ & 3.02 & 2.56 & $2.10^{\mathrm{a}}$ & $9.07^{\mathrm{ab}}$ & $7.43^{\mathrm{bc}}$ & 1.59 & $4.08^{\mathrm{ab}}$ \\
\hline & & D.E. & 0.12 & 0.04 & 0.08 & 1.11 & 0.35 & 0.06 & 0.05 & 0.05 & 0.15 & 0.27 & 0.04 & 0.10 \\
\hline & & Mín & 8.84 & 3.00 & 6.20 & 19.30 & 4.10 & 2.91 & 2.49 & 2.01 & 8.85 & 6.73 & 1.52 & 3.95 \\
\hline & & Máx & 9.35 & 3.18 & 6.55 & 23.10 & 5.33 & 3.12 & 2.70 & 2.17 & 9.40 & 7.85 & 1.67 & 4.25 \\
\hline & 153 & $P$ & 0.0025 & 0.1368 & 0.0471 & 0.2764 & 0.0001 & 0.9298 & 0.0945 & 0.0001 & 0.0001 & 0.0006 & 0.5569 & 0.0007 \\
\hline
\end{tabular}

Longitud del ala anterior derecha (V1), Ancho del ala anterior derecha (V2), Longitud del ala posterior derecha (V3), Número de hámulus del ala posterior (V4), Longitud de la trompa (V5), Longitud de la tibia pata posterior (V6), Longitud del fémur pata posterior (V7), Ancho del cuarto tergito (V8), Longitud del cuarto tergito (V9), Longitud banda cuarto tergito (V10), Ancho del cuarto esternito (V11), y Longitud del cuarto esternito (V12).

$P=$ valores $\leq 0.05$, indican diferencias significativas; $\mathrm{D} . \mathrm{E}=$ desviación estándar. 
En la gráfica de influencias (Figura 1) se muestran los coeficientes de cada variable de los dos primeros componentes, siendo la longitud del ala anterior derecha (V1 LAAD), longitud del ala posterior derecha (V3 LAPD), longitud del fémur pata posterior (V7 LFPP), ancho del cuarto tergito (V8 ACT), longitud del cuarto tergito (V9 LCT) y longitud del cuarto esternito (V12 LCE) las variables con mayor influencia sobre CP1. Sin embargo, las tres variables discriminantes explican solamente el $60.088 \%$ de la variabilidad de los datos originales, lo cual se considera insuficiente para los fines del presente análisis.

Figura 1: Información bidimensional de influencias de las variables morfométricas sobre dos principales componentes

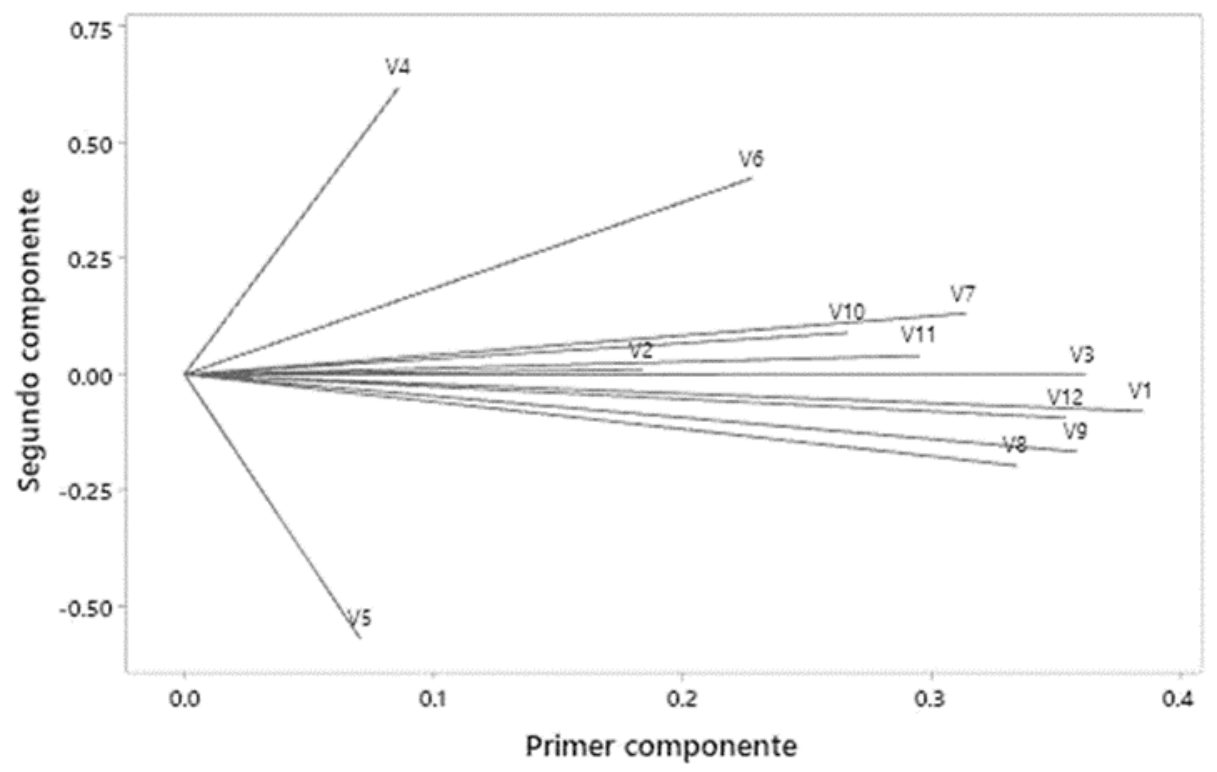

Longitud del ala anterior derecha (V1), Ancho del ala anterior derecha (V2), Longitud del ala posterior derecha (V3), Número de hámulus del ala posterior (V4), Longitud de la trompa (V5), Longitud de la tibia pata posterior (V6), Longitud del fémur pata posterior (V7), Ancho del cuarto tergito (V8), Longitud del cuarto tergito (V9), Longitud banda cuarto tergito (V10), Ancho del cuarto esternito (V11), y Longitud del cuarto esternito (V12).

De acuerdo con el diagnóstico mediante el método FABIS, a nivel estatal ( $\mathrm{n}=153)$, se determinaron 67 colonias $(43.79 \%)$ con morfotipo africanizado, 77 colonias $(50.33 \%)$ con morfotipo europeo y 9 colonias $(5.88 \%)$ sospechosas (S), mostrando diferencias significativas $\left(\mathrm{Ji}^{2}=52.86, \mathrm{n}=153, P=0.0001\right)$. Las colonias sospechosas fueron localizadas en apiarios de la subregión Centro $(\mathrm{n}=31) 1$ colonia $(3.2 \%)$, subregión Chontalpa $(\mathrm{n}=68) 6$ colonias $(8.82 \%)$ y en la subregión Pantanos $(\mathrm{n}=24), 2$ colonias $(8.33 \%)$.

De acuerdo con el análisis del ADNmt (Figura 2, Cuadro 2), a nivel estatal (n=153), se determinaron 86 colonias (56.21\%) con mitotipo Africanizado y 67 colonias $(43.79 \%$ ) con mitotipo Europeo, sin encontrar significancia estadística $\left(\mathrm{Ji}^{2}=2.36, \mathrm{n}=153, P=0.124\right)$. De 
manera específica de las 135 colonias comerciales muestreadas, se detectaron frecuencias relativas de genes del mitotipo E en 52 colonias (38.52\%) y 83 colonias $(61.48 \%)$ presentaron mitotipo A encontrando diferencias significativas $\left(\mathrm{Ji}^{2}=7.12, \mathrm{n}=135, P=0.0076\right)$. Lo cual demuestra el predominio del mitotipo africanizado sobre el europeo en un orden de $1.59 \mathrm{~A} / \mathrm{E}$ en las colonias comerciales. Como puede observarse en el Cuadro 2, en promedio, la subregión de los Ríos mostró la frecuencia más baja de mitotipo A (41.67 \%), encontrándose mayor grado de africanización en el resto de las subregiones, hasta $75 \%$ en la subregión Pantanos.

Figura 2: Amplificación del ADNmt de Apis mellifera digerido con la enzima de restricción $B g l I I$

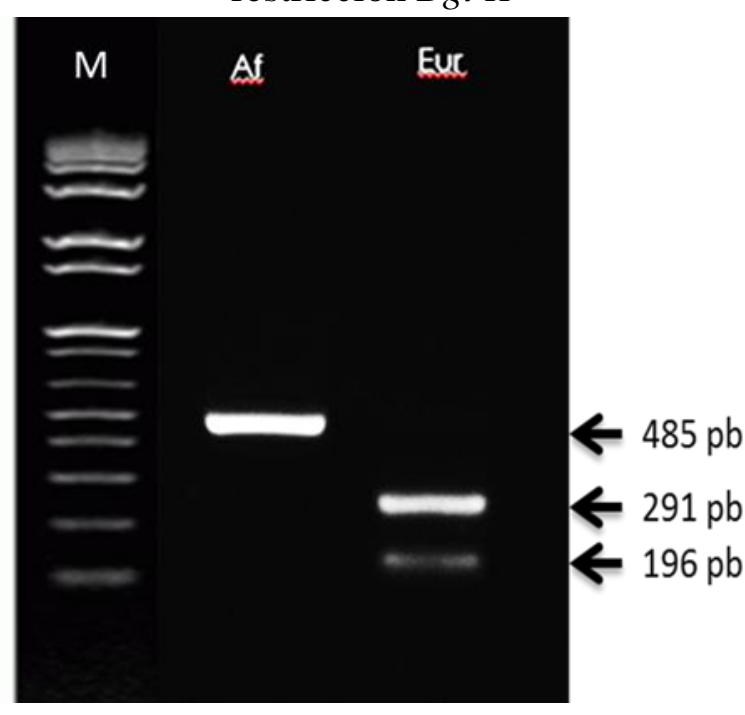

Af = Africanizado, mitotipo sin digerir, Eur= Europeo (doble bandeo). $\mathrm{M}=$ marcador de talla molecular.

Al realizar el análisis morfométrico de las 12 variables empleando como fuentes de variación las cuatro categorías descritas, se encuentran diferencias estadísticamente significativas en el $67 \%$ de las variables ( 8 de 12). En el Cuadro 3, puede observarse que existen pequeñas diferencias numéricas, sin embargo, las abejas E-CPC presentan en la mayoría de las variables morfométricas las dimensiones más grandes, excepto en V5 LTR, sin embargo, no existen diferencias significativas. 
Cuadro 2. Número y proporción (\%) de colonias clasificadas mediante FABIS y el mitotipo del ADNmt, identificado como africanizado - europeo en las subregiones del estado de Tabasco

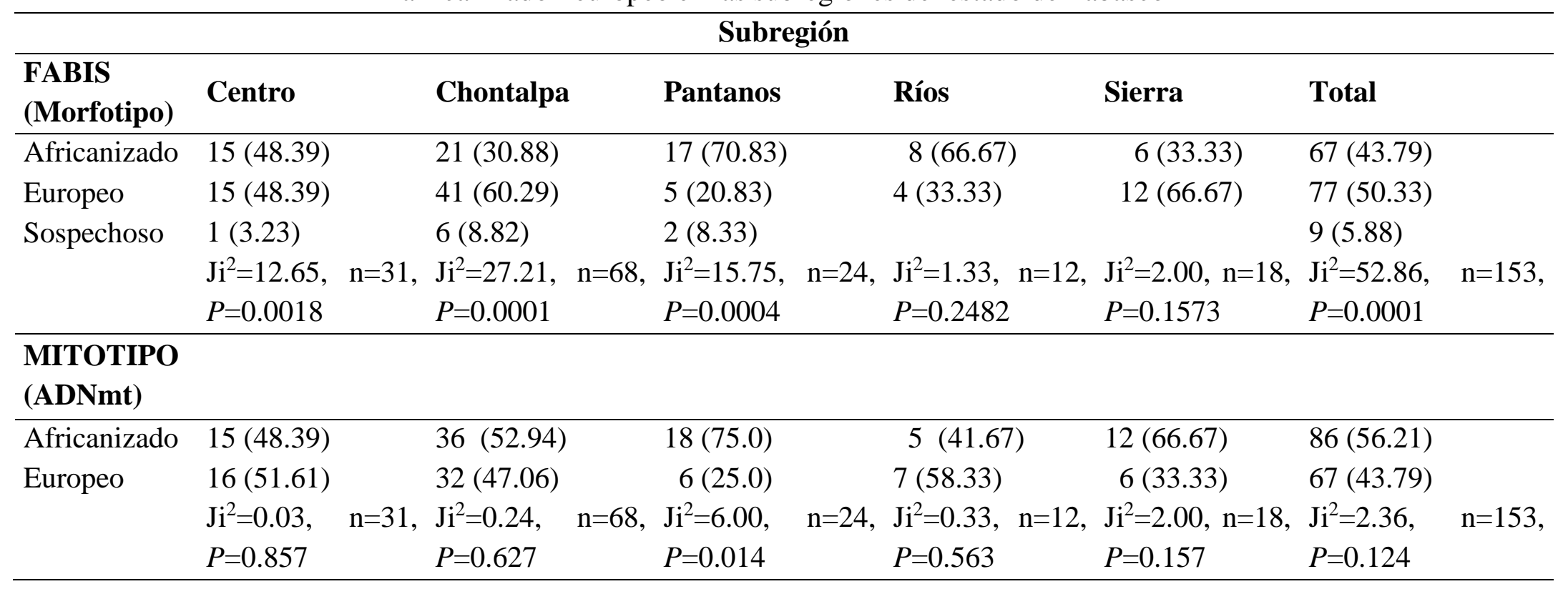

$P=$ valores de $\leq 0.05$, indican diferencias significativas. 
Cuadro 3: Análisis morfométrico entre abejas con mitotipo: $\mathrm{A}-\mathrm{CC}=$ Africanizado colonia comercial, $\mathrm{A}-\mathrm{CS}=\mathrm{Africanizado}$ colonia silvestre, E-CC= Europeo colonia comercial y E-CPC= Europeo colonia pie de cría $(\mathrm{mm})$

\begin{tabular}{|c|c|c|c|c|c|c|c|c|c|c|c|c|c|c|}
\hline Categorías & $\mathbf{n}$ & Resumen & $\mathrm{V1}$ & $\mathrm{V2}$ & V3 & $\mathrm{V4}$ & $\mathrm{V5}$ & V6 & V7 & V8 & V9 & V10 & V11 & V12 \\
\hline \multirow{5}{*}{$\mathrm{A}-\mathrm{CC}$} & \multirow{4}{*}{83} & Media & $8.99^{b}$ & $3.07^{\mathrm{ab}}$ & $6.31^{b}$ & 21.08 & 5.03 & 3.00 & $2.55^{\mathrm{ab}}$ & $2.07^{\mathrm{b}}$ & $8.95^{b}$ & 7.36 & $1.60^{\mathrm{b}}$ & $4.05^{b}$ \\
\hline & & D.E. & 0.12 & 0.09 & 0.10 & 0.95 & 0.38 & 0.12 & 0.05 & 0.06 & 0.19 & 0.28 & 0.04 & 0.11 \\
\hline & & Mínima & 8.71 & 2.92 & 6.06 & 18.4 & 4.10 & 2.10 & 2.44 & 1.84 & 8.47 & 6.50 & 1.50 & 3.77 \\
\hline & & Máxima & 9.36 & 3.76 & 6.59 & 23.50 & 5.65 & 3.19 & 2.69 & 2.36 & 9.40 & 7.96 & 1.69 & 4.29 \\
\hline & \multirow{4}{*}{3} & Media & $8.74^{\mathrm{c}}$ & $3.0^{\mathrm{b}}$ & $6.07^{\mathrm{c}}$ & 20.83 & 5.12 & 2.93 & $2.53^{\mathrm{b}}$ & $1.98^{\mathrm{b}}$ & $9.01^{\mathrm{ab}}$ & 7.23 & $1.50^{\mathrm{c}}$ & $3.91^{\mathrm{b}}$ \\
\hline \multirow{4}{*}{ A-CS } & & D.E. & 0.09 & 0.06 & 0.07 & 0.47 & 0.20 & 0.03 & 0.03 & 0.05 & 0.57 & 0.12 & 0.04 & 0.06 \\
\hline & & Mínima & 8.64 & 2.94 & 5.99 & 20.30 & 4.90 & 2.91 & 2.50 & 1.93 & 8.63 & 7.09 & 1.48 & 3.85 \\
\hline & & Máxima & 8.82 & 3.07 & 6.13 & 21.20 & 5.26 & 2.96 & 2.56 & 2.03 & 9.67 & 7.32 & 1.55 & 3.97 \\
\hline & \multirow{4}{*}{52} & Media & $9.08^{\mathrm{ab}}$ & $3.09^{\mathrm{a}}$ & $6.36^{\mathrm{ab}}$ & 21.01 & 5.09 & 3.02 & $2.57^{\mathrm{ab}}$ & $2.08^{\mathrm{ab}}$ & $9.00^{\mathrm{ab}}$ & 7.45 & $1.60^{\mathrm{b}}$ & $4.07^{\mathrm{ab}}$ \\
\hline \multirow{4}{*}{$\mathrm{E}-\mathrm{CC}$} & & D.E. & 0.17 & 0.06 & 0.12 & 0.83 & 0.38 & 0.09 & 0.07 & 0.06 & 0.24 & 0.33 & 0.05 & 0.14 \\
\hline & & Mínima & 8.80 & 2.98 & 6.10 & 19.60 & 4.38 & 2.83 & 2.39 & 1.88 & 8.54 & 6.54 & 1.50 & 3.61 \\
\hline & & Máxima & 9.49 & 3.32 & 6.64 & 22.80 & 5.77 & 3.22 & 2.80 & 2.23 & 9.66 & 8.22 & 1.71 & 4.37 \\
\hline & \multirow{4}{*}{15} & Media & $9.20^{\mathrm{a}}$ & $3.12^{\mathrm{a}}$ & $6.43^{\mathrm{a}}$ & 21.45 & 4.96 & 3.04 & $2.60^{\mathrm{a}}$ & $2.12^{\mathrm{a}}$ & $9.14^{\mathrm{a}}$ & 7.52 & $1.64^{\mathrm{a}}$ & $4.15^{\mathrm{a}}$ \\
\hline \multirow{4}{*}{ E-CPC } & & D.E. & 0.11 & 0.05 & 0.11 & 1.130 & 0.45 & 0.05 & 0.06 & 0.04 & 0.17 & 0.34 & 0.04 & 0.08 \\
\hline & & Mínima & 9.06 & 3.02 & 6.28 & 19.90 & 4.18 & 2.98 & 2.50 & 2.04 & 8.79 & 6.73 & 1.57 & 4.03 \\
\hline & & Máxima & 9.41 & 3.19 & 6.66 & 23.80 & 5.58 & 3.13 & 2.70 & 2.19 & 9.48 & 8.15 & 1.72 & 4.33 \\
\hline & 153 & $P$ & 0.0001 & 0.0238 & 0.0001 & 0.3993 & 0.6237 & 0.2754 & 0.0222 & 0.0019 & 0.0198 & 0.1201 & 0.0001 & 0.0027 \\
\hline
\end{tabular}

Longitud del ala anterior derecha (V1), Ancho del ala anterior derecha (V2), Longitud del ala posterior derecha (V3), Número de hámulus del ala posterior (V4), Longitud de la trompa (V5), Longitud de la tibia pata posterior (V6), Longitud del fémur pata posterior (V7), Ancho del cuarto tergito (V8), Longitud del cuarto tergito (V9), Longitud banda cuarto tergito (V10), Ancho del cuarto esternito (V11), y Longitud del cuarto esternito (V12); D.E.= desviación estándar. $P=$ valores $\leq 0.05$, indican diferencias significativas.

${ }^{\mathrm{ab}}$ Literales diferentes en la misma columna indican diferencias significativas. 
El análisis de agrupamiento presentado en la Figura 3, muestra que, en la primera etapa del procedimiento, la categoría A-CC se agrupa con la categoría E-CC (1.63). Seguidamente se forma un segundo grupo con la categoría E-CPC con una distancia de agrupamiento de 3.96, apareciendo la categoría A-CS como el grupo más alejado (5.76).

Figura 3: Fenograma obtenido por el método de agrupamiento del vecino más cercano de 12 variables morfométricas, medidas en abejas meliferas (Apis mellifera $\mathrm{L}$.) en el estado de Tabasco

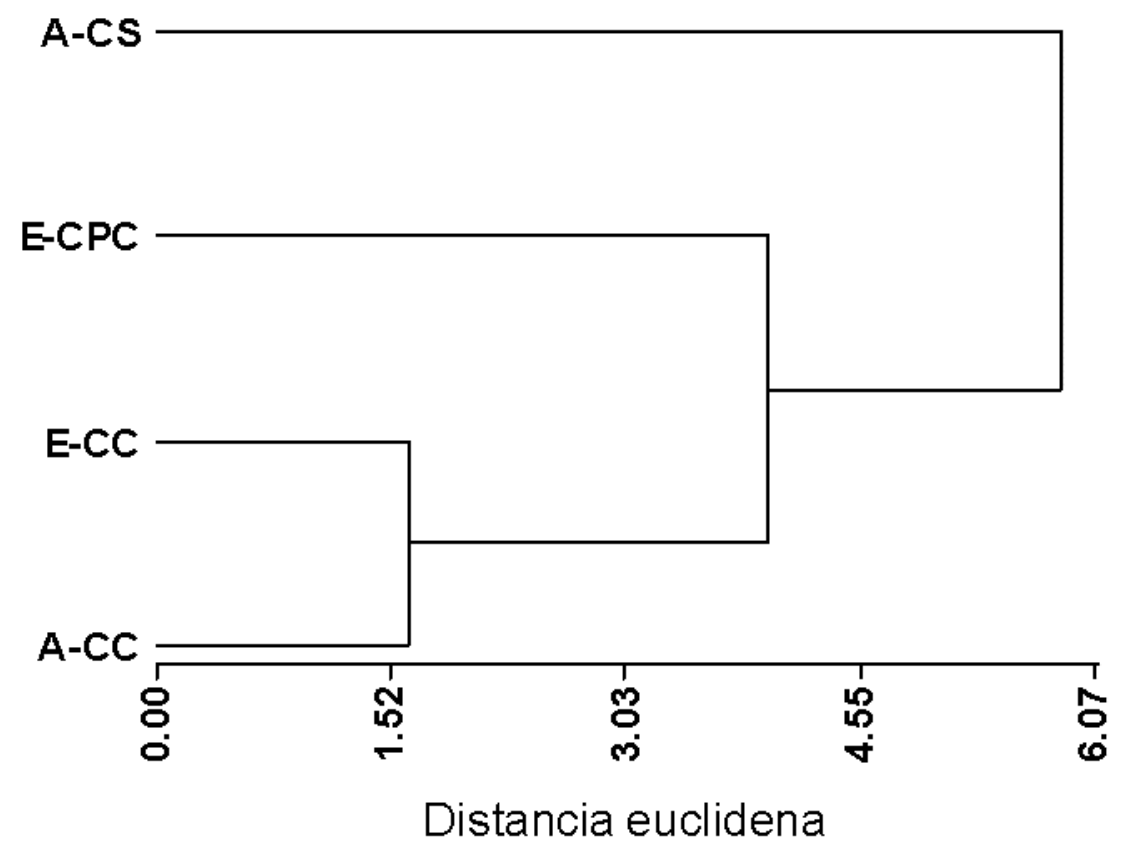

Discusión

$\mathrm{Al}$ emplear las subregiones como fuentes de variación, las diferencias encontradas en las variables de longitud y ancho de las estructuras, aunque significativas son muy estrechas y no es posible lograr una discriminación clara de las abejas entre las subregiones. En promedio las abejas del estado de Tabasco, miden V1 LAAD (9.022 mm), V2 AAAD (3.13 mm), V3 LAPD $(6.326 \mathrm{~mm})$, V4 NHAP (cuentan con 21.012 hámulos), V6 LTPP (3.01 mm), inferiores a las abejas criollas de una población de abejas africanizadas Apis mellifera sp de la región Lambayeque en Perú( ${ }^{(25)}$. No obstante, las abejas de Tabasco son más grandes en cuanto a V7 LFPP (2.56 mm).

Las correlaciones momento producto de Pearson, entre cada par de variables ( $\mathrm{n}=153)$, mostró que la V1 LAAD tiene fuerte correlación positiva y significativa con V2 AAAD (0.5072), 
V3 LAPD (0.8368), V7 LFPP (0.5220), V8 ACT (0.5377), V9 LCT (0.5568) y V12 LCE (0.5502), pero con el resto las correlaciones son débiles. Llama la atención la débil correlación (0.1541) no significativa entre V3 LAPD y V4 NHAP. La V5 LTR, solo tiene correlación débil positiva significativa con V9 LCT (0.1754), su correlación con el resto de las variables es débil y no significativo. La V7 LFPP, mostró correlación significativa con V8 ACT (0.4130), V9 LCT (0.4785), V10 (0.2910), V11 (0.4719) y V12 (0.4550). Con relación al tergito y esternito, las medidas, correlacionan positiva y significativamente entre sí. La V8 ACT, tiene fuerte correlación positiva y significativa con V9 LCT (0.6392), V10 (0.3441), V11 (0.4528) y V12 (0.5667).

Se encontraron diferencias significativas tanto entre las subregiones como entre las categorías, en las variables V1 LAAD y V7 LFPP, lo cual tiene especial atención ya que son utilizadas, para la determinación de la africanización mediante el método FABIS ${ }^{(22,23)}$. Así mismo, estas variables en conjunto mostraron correlaciones positivas significativas con 11 de las 12 variables estudiadas. Respecto al número de hámulus, en un análisis comparativo entre abejas africanizadas y europeas, se reportó que entre las abejas obreras africanizadas y europeas, estadísticamente no existen diferencias ${ }^{(26)}$; los resultados de este trabajo también confirman esa condición al no encontrar el número de hámulus como una característica discriminante entre ambas razas. En relación a que el patrón morfométrico de las abejas africanas es más pequeño, y que las europeas son más grandes ${ }^{(3,27,28)}$, bajo las condiciones de este trabajo se encontró que si bien, la características morfométricas son diferentes estadísticamente, la diferencia en promedio es muy estrecha, lo cual concuerda con diversos investigadores que afirman que las abejas africanizadas son muy difíciles de diferenciar morfométricamente, y que en la actualidad se tienen abejas africanizadas con características más europeas o viceversa ${ }^{(29,30)}$. Esta similitud morfométrica podría llevar a conclusiones erróneas al emplear métodos morfométricos para la determinación A-E. Lo anterior fue demostrado en poblaciones de abejas morfométricamente africanizadas ${ }^{(31)}$ y concuerda con los resultados en este studio, al encontrar de manera general entre ambos métodos de diagnóstico (FABIS/ADNmt) 59 (38.56 \%) determinaciones no coincidentes. Estos resultados sugieren la posibilidad de encontrar que abejas de mitotipo y sitio de colecta diferente, compartan el mismo grupo morfométrico, por lo que no es posible realizar una clasificación por subregión geográfica de las abejas del estado de Tabasco, lo cual podría explicarse por los usos y costumbres de distribución de abejas reinas entre los productores de diferentes subregiones.

El tamaño corporal de las abejas, tiene una fuerte base genética, sin embargo, se ha demostrado que el manejo de las colonias, particularmente el tamaño de la celda influye sobre el tamaño corporal de las abejas ${ }^{(32)}$. Otro estudio que refuerza la idea de que las prácticas apícolas inciden en el tipo genético de las poblaciones de abejas es el reportado en 2007 por 
Antonio $^{(33)}$, quien encontró que el $67.39 \%$ de las colonias de la comarca lagunera, resultaron europeas, lo cual fue atribuido a la intensidad con la que los apicultores han realizado el reemplazo de reinas. Más recientemente otros investigadores ${ }^{(34)}$ mediante FABIS reportaron que $91.49 \%$ de las colonias de abejas de Mexicali y $67.65 \%$ de las colonias de Ensenada tienen morfotipos africanizados, sin embargo, en los colonias silvestres se encontró que $100 \%$ en Mexicali y $50 \%$ en Ensenada tienen morfotipos africanizados, lo cual coincide con lo encontrado en este trabajo, y que podría ser explicado por las diferencias en la tecnificación de los apiarios muestreados.

El hecho de que en el territorio tabasqueño las abejas con mitotipo A y E en las colonias comerciales tengan características morfométricas muy similares, podría explicarse a) por la hibridación natural con las colonias silvestres desde el ingreso de la abeja africana; b) por la introducción de abejas reinas comerciales o abejas pie de cría europeas; c) influenciado por las características de los panales. La procedencia del mitotipo E en el estado de Tabasco, es diversa, ya que durante 2003 a 2010, el ISPROTAB (Instituto de Sistemas de Producción del Trópico Húmedo de Tabasco); adquirió de criaderos certificados, abejas inseminadas de diferentes razas europeas, para reproducirlas en sus criaderos de reinas y donar a los productores reinas F1, con la finalidad de fomentar el recambio anual de abejas reinas; sin embargo, posterior a su cancelación, algunos apicultores adquieren por su cuenta, abejas reinas, procedentes de criaderos locales o foráneos los cuales pudieran estar o no, certificados por la SAGARPA (Secretaría de Agricultura, Ganadería, Desarrollo Rural, Pesca y Alimentación), con el consecuente riesgo sanitario, por lo que aunado a la presencia de colonias silvestres son la principal fuente de genes de mitotipo A, que poseen una gran capacidad para multiplicarse, y eventualmente la fecundación de reinas vírgenes de mitotipo E, con zánganos africanizados, podrían estar resultando en la proliferación de genes africanizados toda vez que se ha reportado que los genes africanos son dominantes ${ }^{(11)}$.

Los resultados demuestran que los apiarios se componen de colonias con ambos mitotipos en diferentes proporciones, lo cual confirma la coexistencia de ambos tipos raciales de abejas en algunos apiarios, y coincide con lo informado recientemente en un estudio mediante ADNmt realizado en siete zonas de Buenos Aires, Argentina, para un total de 430 colonias, encontrando que colonias derivadas de abejas africanas coexisten con europeas en dos de las siete zonas, además de que los mitotipos europeos continúan siendo más frecuentes, en comparación a los resultados que ellos obtuvieron en $2005^{(35)}$. Por otro lado Quezada-Euan ${ }^{(8)}$ mediante análisis de aloenzimas en 25 colonias manejadas reportó el $95 \%$ de haplotipos AHB (Por sus siglas en inglés Africanized Honeybee) en los estados de Chiapas y Tabasco, y $73 \%$ en Yucatán, encontrando los niveles más bajos en los estados de Michoacán y Jalisco (56 y $40 \%$ respectivamente), lo cual es muy cercano a lo que se encontró en este trabajo (56.21\%), y menciona que sus hallazgos, podrían estar relacionados con la intensidad de las 
prácticas de cambio de reinas. Con relación a estos resultados se reportó el $56.21 \%$ de mitotipos africanizados encontrados en colonias comerciales en el estado de Tabasco, lo cual está abajo del rango reportado en un estudio de la africanización mediante ADNmt realizado en cinco poblaciones del estado de Veracruz México y tres poblaciones de referencia, ya que se detectó del 60 al $77 \%$ de mitotipo africanizado en las poblaciones ubicadas entre 72 a $1,300 \mathrm{msnm}^{(20)}$.

Las dos técnicas de diagnóstico usadas en este trabajo definen claramente la raza de las muestras de los grupos CPC y CS, en las que ambas técnicas coinciden plenamente en los resultados, lo que no es de extrañar para la técnica de ADNmt, sabiendo de su robustez; pero también dan evidencia de que el análisis morfométrico es capaz de definir la raza de las abejas, cuando éstas poseen medidas claramente diferenciables o extremas; sin embargo, cuando las mediciones están en rangos compartidos entre las razas, el análisis morfométrico pierde precisión y los resultados pueden ser erráticos, así lo demuestran las coincidencias y no coincidencias de ambos métodos diagnósticos.

En este sentido es importante mencionar que el análisis de ADNmt manifiesta el origen ancestral de las abejas, por la estabilidad generacional del ADNmt, pero el análisis morfométrico refleja la dificultad de definir racialmente, ya que las poblaciones de abejas se están entrecruzando y poseen características físicas y de comportamiento de ambas razas. En una investigación realizada en Colombia, sin hacer referencia al genotipo de las abejas, se reportó que en las regiones apícolas Tolima y Boyacá, los valores promedio del ala anterior fueron de $8.74 \mathrm{~mm}$ a $8.63 \mathrm{~mm}$, lo cual indica uniformidad morfológica africana o muy cercana a las africanas, por el proceso de africanización de las abejas de esas regiones ${ }^{(9)}$. De igual forma en Colombia, se reportó un patrón de amplificación de sitios Dra I, 16S RFLP, del $87.5 \%$ de colonias africanas (Apis mellifera scutellata) y $12.5 \%$ europeas (cárnica/ligústica), puntualizando que Tolima posee predominantemente genes africanizados ${ }^{(36,37)}$. Respecto a la longitud del ala, se encontró que en las abejas A-CS, A-CC y E-CC, miden en promedio $8.74 \mathrm{~mm}, 8.99 \mathrm{~mm}$ y $9.08 \mathrm{~mm}$ respectivamente. Sin embargo, las abejas con mitotipo E-CC de Tabasco tienen una longitud de ala anterior derecha de 9.08 $\mathrm{mm}$ ligeramente menor que las del altiplano $\geq 9.12 \mathrm{~mm}$ reportadas ${ }^{(19)}$.

La caracterización molecular basada en el ADNmt se ha convertido en una técnica ampliamente utilizada para el estudio de la diferenciación de subespecies o razas de la abeja de la miel Apis mellifera L. por diversos autores ${ }^{(37-40)}$. Esta molécula circular y de herencia materna, permite caracterizar la abeja reina a través de las obreras y así a toda la colonia, pudiendo considerarse un marcador de toda la colonia. Su estudio ha permitido elaborar diferentes hipótesis sobre la evolución de las subespecies de Apis mellifera y definir cinco linajes evolutivos: el linaje A, incluye las subespecies africanas como intermissa y scutellata, 
el linaje M está formado por las subespecies de Europa Occidental, incluyendo mellifera, el linaje $\mathrm{C}$ formado por las subespecies de Europa Oriental (ligustica o abeja italiana), el linaje O que abarca a las subespecies de Oriente Próximo y el Y que incluye a la subespecie Apis mellifera yemenitica de Etiopía ${ }^{(15)}$. Cada uno de estos linajes presenta una composición característica en la secuencia de diferentes regiones del ADN mitocondrial, como sucede con la subunidad I del gen de la citocromo oxidasa (COI), para la que el linaje M presenta una diana de la endonucleasa Hinc II que no se encuentra en el linaje $\mathrm{A}^{(40)}$.

\section{Conclusiones e implicaciones}

Los análisis morfométricos solamente fueron discriminantes en los valores extremos, y por lo tanto clasifican como sospechosas de ser africanizadas, con lo que se podría estar reflejando la realidad de la dinámica entrecruzamientos de las poblaciones. La coexistencia de abejas de tipo europeo y africanizado, ha sido afectada por algunas prácticas como el cambio de reinas, que han influido sustancialmente en el tipo de abejas presentes en las colonias. El comportamiento reproductivo de las abejas melíferas contribuye fuertemente en el mantenimiento de los niveles de africanización de las colonias, ya que permite la introgresión de genes silvestres a las poblaciones de abejas manejadas por los apicultores, lo cual se puede apreciar en las colonias de abejas comerciales identificadas en este trabajo como (CC). Estos resultados discriminan clara y significativamente la categoría A-ES de la E-CPC, mostrando los valores morfométricos extremos; sin embargo, las categorías A-CC y E-CC, presentaron marcadas similitudes; este amplio rango morfométrico, sugiere diversidad genética, la cual debe de ser investigada para determinar los linajes a los que pertenecen las abejas en esta región del país. Hasta el momento el mejor método para discriminar genotipos E o A es el método molecular.

\section{Agradecimientos}

Al Tecnológico Nacional de México por el financiamiento otorgado al proyecto: Determinación de varroa y detección molecular de Nosema apis y Nosema ceranae, en apiarios comerciales de Apis mellifera, en el estado de Tabasco, México. Clave: 6328.17P. 


\section{Literatura citada:}

1. Ruttner F. Biogeography and taxonomy of honey bees. Germany, New York, USA: Springer Verlag: Heiddelberg; 1988.

2. Padilla-Álvarez F, Sereno FTP de S. Estudio de la diversidad morfológica existente en las abejas melíferas (Apis mellífera L.) del sur de Europa y del Continente Sudamericano. Arch Zootec 2005;(54):221-226.

3. Kerr WE. The history of the introduction of Africanized bees in Brazil. S Afr Bee J 1967;(39):3-5.

4. Schneider SS, DeGrandi-Hoffman G, Smith DR. The African honey bee: Factors contributing to a successful invasion. Ann Rev of Entom 2004;(49):351-376. doi:10.1146/annurev.ento.49.061802.123359.

5. Fierro MM, Barraza A, Maki DL, Moffet JO. The effect of the first year of africanization on honey bee populations in Chiapas, Mexico. Proc Third Am Bee Res Conf. Weslaco, Tx. USA. 1987.

6. Moffett JO, Dale LM, Thomas A, Fierro MM. The africanized bee in Chiapas, Mexico. Am Bee J 1987;127(7):517-519.

7. Herrera SA. Monitoreo de abejas africanas. III Seminario Americano de Apicultura. Acapulco, México. 1989;9-11.

8. Quezada-Euan JJG. The present status of african-derived honeybees in tropical México. VI Encontro sobre Abelhas. Riberao Preto Brasil. 2006.

9. Salamanca GG, Vargas EF, Pérez FC. Estudio morfométrico y sistemático del grado de africanización de la abeja. 1999. https://www.apiservices.biz/es/articulos/ordenar-porpopularidad/716-estudio-morfometrico-y-sistematico-del-grado-de-africanizacion.

Consultado 8 Mar, 2019.

10. Danka RG, Hellmich RL II, Rinderer TE, Collins AM. Diet-selection ecology of tropically and temperately adapted honey bees. Anim Behav 1987;(35):1858-1863.

11. Guzmán-Novoa E, Correa-Benítez A, Guzmán G, Espinosa LG. History, colonization and impact of the africanized honey bee in Mexico. Vet Méx 2011;42(2):149-178.

12. FAOSTAT. Base de datos del mundo sobre estadísticas alimentarias y agrícolas. Organización de las naciones unidas. http://www.fao.org/faostat/es/\#rankings/countries_by_commodity_exports. Consultado 15 Feb, 2021. 
13. INFOSIAP. Servicio de información y estadística agroalimentaria. http://infosiap.siap.gob.mx/repoAvance_siap_gb/pecAvanceEdo.jsp. Consultado 15 Feb, 2021.

14. Winston ML. Killer bees: The africanized honey bee in the Americas. Cambridge: Harvard University Press; 1992.

15. Franck P, Garnery L, Loiseau A, Oldroyd BP, Hepburn HR, Solignac M. Genetic diversity of the honeybee in Africa: microsatellite and mitochondrial data. Heredity $2001 ;(86): 420-430$.

16. Doyle JJ, Doyle JL. Isolation of plant DNA from fresh tissue. Focus 1990;12(1):13-16.

17. Crozier YC, Koulianus S, Crozier RH. An improved test for africanized honey bee mitocondrial DNA. Experientia 1991;(47):968-969.

18. Clarke KE, Oldroyd BP, Javier J, Quezada-Euan G, Rinderer TE. Origin of honeybees (Apis mellifera $\mathrm{L}$ ) from Yucatan Peninsula inferred from mitocondrial DNA analysis. Mol Ecol 2001;(10):1347-1355.

19. Uribe-Rubio JL, Guzmán-Novoa E, Hunt GJ, Correa-Benítez A, Zozaya JA. Efecto de la africanización sobre la producción de miel, comportamiento defensivo y tamaño de las abejas melíferas (Apis mellifera L.) en el altiplano mexicano. Vet Mex 2003;34(1):4759.

20. Kraus FB, Franck P, Vandame R. Asymmetric introgression of African genes in honeybee populations (Apis mellifera L.) in Central Mexico. Heredity 2007:1-8.

21. de la Rua P, Hernández-García R, Pedersen BV, Galián J, Serrano J. Molecular diversity of honeybee Apis mellifera Iberica L. (Hymenoptera: Apidae) from western Andalusia. Arch Zootec 2004;(53):195-203.

22. Sylvester HA, Rinderer TE. Fast africanized bee identification system (FABIS) manual. Am Bee J 1987;127(7):511-516.

23. INAFED. Instituto Nacional para el Federalismo y el Desarrollo Municipal. Enciclopedia de los municipios y delegaciones de México. 2018. EN: http://www.inafed.gob.mx/work/enciclopedia/emm27tabasco/regionalizacion.html. Consultado 8 May, 2020. 
24. DOF. Diario Oficial de la Federación. Norma oficial mexicana NOM-056-ZOO-1995. Especificaciones técnicas para las pruebas diagnósticas que realicen los laboratorios de pruebas aprobados en materia zoosanitaria. http://www.dof.gob.mx/nota_detalle.php?codigo=4944688\&fecha=22/02/1999. Consultado 15 May, 2020.

25. Vásquez-Arca, OR, Mestanza-Arca BS, Alarcón-Silva RE. Características morfométricas, comportamiento higiénico y agresividad de abejas criollas Apis mellifera sp. Rev Inv Cult 2016;(1). https://www.redalyc.org/jatsRepo/5217/521753139003/html/index.html. Consultado 15 Jul, 2020.

26. del Lama MA, Gruber CV, de Godóy IC. Heterozigosidade e assimetria do número de hámulos em operárias adultas de Apis mellifera (Hymenoptera, Apidae). Rev Bras Entomol 202;46(4):591-595.

27. Guzman-Novoa E, Page RE, Spangler HG, Erickson EH. A comparison of two assays to test the defensive behaviour of honey bee (Apis mellifera L.). J Apic Res 1999;(38):205209.

28. Zhou T, Huang Z, Yao J, Wang G, Huang S. Los efectos que el tamaño de la celda de cría tiene sobre el comportamiento reproductivo de la varroa. China: Apimondia 2010.

29. May-Itzá WJ, Quezada-Euan JJG, Iuit L, Echazarreta CM. Do morphometrics and allozymes reliably distinguis africanized and european Apis mellifera drones in subtropical Mexico?. J Apic Res 2001;40(1):17-23.

30. Pérez-Castro EE, May-Itzá WJ, Quezada-Euan JJG. Thirty years alter: a survey on the distribution and expansion of africanized honey bees (Apis mellifera) in Peru. J Apic Res 2002;41(3-4):69-73.

31. Quezada-Euan JJG, Hinsull SM. Evidence of continued European morphometrics and mtDNA in feral colonies of honey bees (Apis mellifera) from the Yucatan peninsula, Mexico. J Apic Res 1995;34(3):16-166.

32. Masaquiza-Moposita DA, Curbelo RLM, Díaz MB, Arenal CA. Relaciones entre producción melífera, defensividad y diámetro de celdas de cría de Apis mellifera L; en el altiplano ecuatoriano. Rev Prod Anim 2019;31(3). https://revistas.reduc.edu.cu/index.php/rpa/article/view/e2994. Consultado 22 Jun, 2020. 
33. Antonio GMA. Estado actual de la africanización de las abejas melíferas en la Comarca Lagunera [Tesis licenciatura]. Torreón, Coahuila, México: Universidad Autónoma Agraria Antonio Narro Unidad Laguna; 2008.

34. Alaniz-Gutiérrez L, Torres-Salado N, Ail-Catzim CE, Velazco-López JL. Frecuencia de morfotipos africanizados y europeos de Apis mellifera en Ensenada y Mexicali, Baja California. Ecosist Recur Agropec 2016;3(9):421-426.

35. Genchi G, Reynaldi FJ, Bravi CM. An update of Africanization in honey bee (Apis mellifera) populations in Buenos Aires, Argentina. J Apic Res 2018. DOI: 10.1080/00218839.2018.1494887. https://doi.org/10.1080/00218839.2018.1494887. Accessed Jun 15, 2020.

36. Prada QCF, Duran TJ, Salamanca GG, del Lama AM. Population genetics of Apis mellifera L (Hymenoptera: Apidae) from Colombia. J Apic Res 2009;48(1):3-10.

37. Garnery L, Cornuet JM, Solignac M. Evolutionary history of the honeybee Apis mellifera L. inferred from mitochondrial DNA analysis. Mol Ecol 1992;(1):145-154.

38. de la Rua P, Galián J, Serrano J. Variabilidad del ADN mitocondrial en poblaciones de abejas de la miel (Apis mellifera L.) de la región de Murcia. Invest Agr Prod Sanid Anim $1999 ; 14(3): 41-49$.

39. de la Rua P, Galián J, Serrano J, Moritz RFA. Molecular characterization and population structure of honey bee from the Belaric island (Spain). Apidologie 2001;(32):417-427.

40. Hall HG, Smith DR. Distinguishing african and european honeybee matrilines using amplified mitochondrial DNA. Proc Natl Acad Sci USA. 1991;88(10):4548-52. 